\title{
BMJ Open Plasma concentration guided dosing of drugs used for the treatment of childhood leukaemias: protocol for a systematic review
}

\author{
Madelé van Dyk (D) , ${ }^{1}$ Chelsea Boylan, ${ }^{2}$ Robin Michelet, ${ }^{3,4}$ \\ Anna M Mc Laughlin (1) , 3,5 Ganessan Kichenadasse, ${ }^{2,6}$ Nikki May, ${ }^{7}$ \\ Victoria Ziesenitz (D) , ${ }^{8}$ Johannes N Van Den Anker, ${ }^{9,10}$ Stefanie L Groenland, ${ }^{11,12}$ \\ Alwin D R Huitema, ${ }^{13,14}$ Neeltje Steeghs, ${ }^{11,12}$ Gerd Mikus, ${ }^{15,16}$ Charlotte Kloft, ${ }^{17}$ \\ Heather Tapp ${ }^{18}$
}

To cite: van Dyk M, Boylan C, Michelet $\mathrm{R}$, et al. Plasma concentration guided dosing of drugs used for the treatment of childhood leukaemias: protocol for a systematic review. BMJ Open 2022;12:e053308. doi:10.1136/ bmjopen-2021-053308

- Prepublication history and additional supplemental material for this paper are available online. To view these files, please visit the journal online (http://dx.doi.org/10.1136/ bmjopen-2021-053308)

Received 10 May 2021 Accepted 19 November 2021

Check for updates

(c) Author(s) (or their employer(s)) 2022. Re-use permitted under CC BY-NC. No commercial re-use. See rights and permissions. Published by BMJ.

For numbered affiliations see end of article.

Correspondence to Dr Madelé van Dyk; madele.vandyk@flinders.edu.au

\section{ABSTRACT}

Introduction Childhood leukaemia is the most common type of cancer in children and represents among $25 \%$ of the diagnoses in children $<15$ years old. Childhood survival rates have significantly improved within the last 40 years due to a rapid advancement in therapeutic interventions. However, in high-risk groups, survival rates remain poor. Pharmacokinetic (PK) data of cancer medications in children are limited and thus current dosing regimens are based on studies with small sample sizes. In adults, large variability in PK is observed and dose individualisation (plasma concentration guided dosing) has been associated with improved clinical outcomes; whether this is true for children is still unknown. This provides an opportunity to explore this strategy in children to potentially reduce toxicities and ensure optimal dosing. This paper will provide a protocol to systematically review studies that have used dose individualisation of drugs used in the treatment of childhood leukaemias.

Methods and analysis Systematic review methodology will be applied to identify, select and extract data from published plasma guided dosing studies conducted in a paediatric leukaemia cohort. Databases (eg, Ovid Embase, Ovid MEDLINE, Ovid Cochrane) and clinical trial registries (CENTRAL, ClinicalTrials.gov and ISRCTN) will be used to perform the systematic literature search (up until February 2021). Only full empirical studies will be included, with primary clinical outcomes (progression-free survival, toxicities, minimal residual disease status, complete cytogenetic response, partial cytogenetic response and major molecular response) being used to decide whether the study will be included. The quality of included studies will be undertaken, with a subgroup analysis where appropriate.

Ethics and dissemination This systematic review will not require ethics approval as there will not be collection of primary data. Findings of this review will be made available through publications in peer-reviewed journals and conference presentations. Gaps will be identified in current literature to inform future-related research. PROSPERO registration number CRD42021225045.

\section{Strengths and limitations of this study}

This review will be the first to summarise available studies regarding dose individualisation of drugs used to treat childhood leukaemias, and how they have been used in clinical practice.

- This review will assess associations between specific chemotherapeutic plasma concentration data and clinical outcomes.

- Our review includes a focus on small molecule targeted therapies, monoclonal antibodies and chemotherapies encompassing many of the current treatment options for childhood leukaemia, thereby forming an up-to-date analysis of treatments available for our study indication.

- This review assesses available information about the associations between clinical outcome data and the pharmacokinetics of drugs used to treat childhood leukaemia and how it is being clinically applied; this type of data are scarce.

\section{INTRODUCTION}

Globally, leukaemia is the most common $(25 \%)$ childhood cancer with the highest incidence in children aged 1-4 years. ${ }^{1}$ In 2018, it was estimated that worldwide more than 29000 childhood cancer deaths were due to leukaemia. $^{2}$ Acute lymphoblastic leukaemia (ALL) is the most common childhood leukaemia; the 5-year survival rate within low risk and standard risk groups has improved to $90 \%$ during the past 40 years due to increased participation in studies, allowing clinicians to build on previous successes. ${ }^{3}$ However, 5-year survival rates within paediatric patients with ALL identified as high risk or very high risk remain between $40 \%$ and 50\%. ${ }^{4}$ Therapies have become more risk stratified with the potential to reduce toxicity and long-term 
sequelae. ${ }^{34}$ For childhood acute leukaemias (ALL and acute myeloid leukaemia; AML) treatments largely consists of protocolised combination pharmacotherapy including standard chemotherapy, targeted therapy and corticosteroids (further detailed in online supplemental appendix 1). For ALL, these therapies are used over the course of 2-3 years. ${ }^{5}{ }^{6}$ For AML the therapy duration is much shorter lasting for approximately 6 months. Small molecule kinase inhibitors are commonly used in specific cancers such as Philadelphia chromosome positive chronic myeloid leukaemia (CML) and ALL. ${ }^{56}$ In addition, bispecific $\mathrm{T}$ cell engagers are now available for the first-line therapy of paediatric patients with ALL and for management of relapse or refractory disease. ${ }^{7}$ Similarly, monoclonal antibodies have now been incorporated into chemotherapeutic regimens to improve outcomes in children with AML. ${ }^{6}$ It is well recognised that these novel treatment regimens may have short-term toxicities ${ }^{7}$ and that long-term effects are still unknown ${ }^{8}$

The accepted practice of paediatric dosing is either by body surface area or weight-based dosing (ie, $\mathrm{mg} / \mathrm{kg}$ ) due to concerns related to the narrow therapeutic index of cytotoxic anticancer drugs and the assumed relationships between body size and drug disposition in these patients. ${ }^{9}$ Many factors that may need to be considered include the maturity of drug metabolising enzyme systems, differences in enzyme activity that may be genetic, the effects of obesity and concomitant medications and diet. ${ }^{10}$ Our rationale for assessing data on plasma concentration guided dosing of drugs used in the treatment of childhood leukaemia include: (1) It is well recognised that pharmacokinetic (PK) data of anticancer drugs in children are extremely limited and thus dosing regimens are often extrapolated from adult data and based on paediatric studies with a small sample size $;^{11} 12$ (2) When administering drugs, there are notable differences in PK and pharmacodynamic properties between adults and children such as age-related differences in the way drugs are absorbed, distributed, metabolised and eliminated; ${ }^{13}$

(3) There is an opportunity to assess the current state of the art for the optimal dosing in paediatric patients with leukaemia ${ }^{14}$ as in adults with leukaemia (eg, imatinib TDM for CML), therapeutic drug monitoring (TDM), using target plasma concentration guided dosing has been demonstrated to optimise exposure and is associated with favourable treatment outcomes (response and survival). ${ }^{15}$ These target concentrations have not been defined for many drugs used for the treatment of leukaemia in children; and (4) In addition, as childhood ALLs require cancer chemotherapy on an ongoing basis for many months, adherence to prescribed therapies may not be consistent or unexpected toxicities may occur with routine dosing. TDM as part of plasma concentration guided dosing provides additional benefits of monitoring for adherence to prescribed therapies and optimising dosing. Furthermore, the relationship between target plasma drug concentration and outcome/toxicity and whether plasma concentration guided dosing will improve the outcome of the treatment has been poorly investigated in childhood leukaemia. Finally, this review will assess the evidence and the quality of the evidence for plasma guided dosing of all drugs used for the treatment of childhood leukaemia.

\section{RESEARCH AIMS AND OBJECTIVES}

This study aims to conduct a systematic review of the approach of using target plasma concentration guided dosing for drugs used to treat childhood leukaemia's.

\section{METHODS AND DESIGN \\ Patient and public involvement}

There will be no patient or public participation involvement as this systematic review is capturing previous findings. However, to increase insight and perspective from people living with cancer, we involve members from our consumer engagement group to provide feedback on our research study design. Therefore, we would like to acknowledge Mr Ryan Hodges, from our consumer engagement group, who have provided verbal feedback on our study design for this protocol paper and will continue that into the systematic literature review (SLR) too.

\section{Inclusion criteria}

- Studies investigating any medications used to treat childhood leukaemias, both approved or off-label (chemotherapy, targeted therapies, monoclonal antibodies,) that report on plasma concentration guided dosing strategies in a paediatric population (0-21 years, including neonates, infants and young children).

- Studies that directly compare monitoring of medications used for the treatment of leukaemia in adult cohorts that are extrapolated to paediatric cohorts.

- Retrospective, prospective, case series, descriptive, quantitative or simulation-based studies reporting plasma concentrations in paediatrics.

- Trial-based or non-trial-based studies, randomised clinical trials or non-randomised controlled studies.

- Studies published in conference abstracts.

- Studies published in the English language.

\section{Exclusion criterion}

- Studies that only included adult populations.

- Studies that are not reporting data on plasma concentrations, (modelling, simulation based, therapeutic drug monitoring, plasma dosing, serum adjusted levels).

- Studies that have a non-clinical experimental design or written as reviews (reviews may be used as a data source to find relevant studies).

- Study will be excluded if it does not relate to the condition or domain being reviewed (childhood 
leukaemia) or does not include a drug therapy used to treat leukaemias.

\section{Condition or domain}

Condition or domain under study is childhood leukaemia.

\section{Population}

Real patients or data simulated from paediatric patients of any sex and race, inpatients or outpatients, who are treated with any antileukaemia agents such as chemotherapies and targeted therapies such as kinase inhibitors and monoclonal antibodies.

\section{Outcome measures}

Relevant primary outcomes will include clinical outcomes such as patient survival (eg, overall survival and relapsefree survival). Where there is opportunity to be more specific, secondary outcomes such as rates of major molecular response (MMR), complete cytogenetic response $(\mathrm{CCyR})$ and partial cytogenetic response $(\mathrm{PCyR})$ in the case of paediatric CML and achievement of minimal residual disease (MRD) negativity in paediatric ALL will also be assessed. Where possible, toxicity data and duration of therapies will also be reported.

\section{Exposures/interventions}

The primary exposure in this review will be plasma concentrations of any kinase inhibitor, monoclonal antibody or chemotherapy used for the treatment of leukaemia in paediatric patients. Any intervention aimed at individualising drug dosage (toxicity adjusted dosing, model-informed precision dosing, genotyping or phenotyping approaches) will also be included as secondary exposures.

\section{Study design}

The systematic review will consider quantitative studies of good quality (based on quality assessment below) published from the databases' inception until February 2021. The searches will be rerun immediately prior to the final analyses and any further studies retrieved will be screened for inclusion.

\section{Search strategies}

The following steps will be undertaken to perform the search strategy. An initial focused search of MEDLINE (PubMed) and Google Scholar will be undertaken. An analysis of the text words contained in the title and abstracts, and the index terms assigned to the results will then be used to develop the Medical Subject Headings $(\mathrm{MeSH})$ and key terms for the search. Four predefined search concepts relating to the research question will be used; these are detailed below:

- Concept 1: will include all MeSH, substance names and key terms for all approved and off-label medications for treating childhood leukaemias.

- Concept 2: will focus on the disease area and will include the MeSH of leukaemia as well as key terms including cancer, leukaemia, oncology and neoplasms.
- Concept 3: will be interventions such as precisionbased dosing. MeSH terms include precision medicine and drug monitoring; additional key terms will include individualised dosing, plasma guided dosing, therapeutic drug monitoring, plasma concentrations and optimal dosing.

- Concept 4: will focus on the patient cohort. MeSH terms include adolescent and child and an extensive set of key terms including paediatric, childhood, neonatal, infant and youth.

A detailed search strategy applied in MEDLINE is provided in the online supplemental appendix 1.

Second, the search will be adapted, using all identified keywords and index terms, specifically for the following databases: Ovid Embase (1974+), Ovid MEDLINE (1946+), Ovid Cochrane (2005+), Ovid EmCare (1995+), EBSCO CINAHL Plus (1936+), Scopus (1996+), ClinicalTrials.gov (2000+) and Web of Science (1945+). Finally, we will undertake backward and forward citation chaining of relevant documents (including Food and Drug administration, Therapeutic Goods Aministration and European Medicines Agency documents).

\section{Study selection}

Titles and abstracts from each database will be screened and relevant records selected for a full-text appraisal. The study selection process will follow the Preferred Reporting Items for Systematic Reviews and Meta-Analyses guidelines. ${ }^{11}$ Search results will be exported into the citation management software EndNote, and into the systematic review software, Covidence. Titles and abstracts will be distributed among three independent reviewers for screening against the inclusion criteria. The strength of agreement between reviewers will be estimated by calculating the intraclass correlation coefficient. ${ }^{16}$ Two reviewers will then assess the full text of selected articles for eligibility. Any disagreement or conflicting views will be resolved by discussion or the final judgement of a third reviewer. Included articles will then progress to quality assessment or critical appraisal, data extraction and analysis.

\section{Quality assessment}

The review will include studies with differences in study design, therefore, the selected papers will be assessed for methodological validity using a Mixed Methods Appraisal Tool. ${ }^{17}$ Studies will not be excluded based on the outcome of the quality assessment as the assessment is aimed to offer general information about the quality and strength of the existing frameworks and evidence of plasma concentration guided dosing of drugs used to treat leukaemia in children.

\section{Data extraction}

Two reviewers will screen the initial articles based on title and abstract in Covidence. The reviewers will independently perform a full-text review on the identified articles against the inclusion and exclusion criteria. The 
data extracted will include specific details about the dosing strategies (ie, standard (one-size fits-all), body weight-based, body surface area-based, plasma concentration guided dosing strategies), the settings, the population and sample size and outcomes as well as details of the results. In the case that the data are not interpretable, citing articles will be explored and if this information is insufficient, the study will be excluded.

\section{Strategy for data synthesis}

Following data extraction, the reviewers will provide a narrative synthesis of the results from the included studies, structured around general characteristics, characteristics of the intervention programmes and treatment endpoints concluded in the study (progression-free survival, overall survival, disease-free survival, relapsefree survival, event-free survival, death, toxicity and disease-specific endpoints such as MMR, CCyR, PCyR and MRD). The statistical analyses of the data conducted by the included studies will also be briefly discussed in this review.

\section{Analysis}

We are interested in the relationship between plasma concentrations (or exposures) of drugs used to treat leukaemias and clinical outcomes in children. Therefore, a narrative synthesis of the outcomes of the selected studies will be presented in the final review. The plasma concentration parameter (eg, minimum plasma concentration: $\mathrm{C}_{\min }$, maximum plasma concentration: $\mathrm{C}_{\max }$ or area under the plasma concentration versus time curve: AUC), control group, sample size, demographic and clinical characteristics and clinical endpoints will be included.

\section{ETHICS AND DISSEMINATION}

This systematic review will not require ethics approval as there will not be any collection of primary data. Findings of this review will be disseminated through publications in peer-reviewed journals, presentations at workshops or conferences and sharing through a media release.

\section{CONCLUSION}

This systematic review will assess and summarise available studies regarding associations between plasma concentration data for drugs used to treat childhood leukaemia, and clinical outcomes. It will specifically review the evidence of plasma concentration guided dosing in children with leukaemia and how they have been used in clinical practice. It will provide support for, or against, the hypothesis that individualised dosing of therapies used to treat childhood leukaemia could improve patient outcomes due to optimised patient dosing and reduction in the rate of adverse events/toxicities.

\section{Author affiliations}

${ }^{1}$ Flinders Health and Medical Research Institute - Cancer, Flinders University, Adelaide, South Australia, Australia
${ }^{2}$ Flinders Health and Medical Research Institute - Cancer, Flinders University College of Medicine and Public Health, Bedford Park, South Australia, Australia

${ }^{3}$ Department of Clinical Pharmacy and Biochemistry, Institute of Pharmacy, Freie Universität Berlin, Berlin, Germany

${ }^{4}$ PharMetrX Graduate Research Training Program, Postdam/Berlin, UK

${ }^{5}$ Postdam/Berlin, PharMetrX Graduate Research Training Program, Postdam/Berlin, Germany

${ }^{6}$ Medical Oncology, Flinders Medical Centre, Bedford Park, South Australia, Australia ${ }^{7}$ SA Health Library Service, Bedford Park, South Australia, Australia

${ }^{8}$ Pediatric Cardiology \& Congenital Heart Diseases, University Hospital Heidelberg, Heidelberg, Germany

${ }^{9}$ Division of Paediatric Pharmacology and Pharmacometrics, University Children's Hospital Basel, Basel, Switzerland

${ }^{10}$ Division of Clinical Pharmacology, Children's National Hospital, Washington,

District of Columbia, USA

${ }^{11}$ Antoni van Leeuwenhoek Netherlands Cancer Institute, Amsterdam, Noord-

Holland, The Netherlands

${ }^{12}$ Department of Medical Oncology, Netherlands Cancer Institute, Amsterdam, Noord-Holland, The Netherlands

${ }^{13}$ Department of Pharmacy \& Pharmacology, Antoni van Leeuwenhoek Netherlands Cancer Institute, Amsterdam, Noord-Holland, The Netherlands

${ }^{14}$ Department of Clinical Pharmacy, University Medical Center Utrecht, Utrecht, The Netherlands

${ }^{15}$ Clinical Pharmacology and Pharmacoepidemiology, UniversitatsKlinikum Heidelberg, Heidelberg, Baden-Württemberg, Germany

${ }^{16}$ Department of Clinical Pharmacy and Biochemistry, Free University of Berlin, Berlin, Germany

${ }^{17}$ Freie Universitat Berlin Institut fur Pharmazie, Berlin, Germany

${ }^{18}$ Haematology/Oncology Unit, Women's and Children's Hospital Adelaide, North Adelaide, South Australia, Australia

\section{Twitter Victoria Ziesenitz @VZieseHD}

Contributors Conception or Design: MvD, RM, CK, NM, SLG, Meuller-Schoell and HT. Acquisition or Analysis of Data: MvD, CB, NM and GK. Interpretation of Data: : MvD, CB, NM, JNVDA, SLG, NS, GM, CK, RM and HT. Drafting the work or revising for intellectual content: MvD, CB, Meuller-Schoell, GK, NM, RM, VZ, JNVDA, ADRH, GM, CK, NS, SLG and HT. Final approval of the version to be published: MvD, CB, RM, AMML, GK, NM, VZ, JNVDA, SLG, ADRH, NS, GM, CK and HT. Agreement to be accountable for all aspects of the work: MvD, CB, RM AMML, GK, NM, VZ, JNVDA, SLG, ADRH, NS, GM, CK and HT.

Funding MvD reports grants from Cancer Council SA/Flinders University (ECR Beat Cancer/10686). CK reports grants from an Industry Consortium (AbbVie Deutschland $\mathrm{GmbH} \& \mathrm{Co}$. KG, AstraZeneca GmbH, Boehringer Ingelheim Pharma GmbH \& Co. KG, Grünenthal GmbH, F. Hoffmann-La Roche Ltd, Merck KGaA and SANOFI) for the PharMetrX programme/grant number: NA, The Federal Ministry of Education/grant number: NA and Research and the European Commission within in the Horizon 2020 framework programme/grant number: NA.

Competing interests None declared.

Patient consent for publication Not applicable.

Provenance and peer review Not commissioned; externally peer reviewed.

Supplemental material This content has been supplied by the author(s). It has not been vetted by BMJ Publishing Group Limited (BMJ) and may not have been peer-reviewed. Any opinions or recommendations discussed are solely those of the author(s) and are not endorsed by BMJ. BMJ disclaims all liability and responsibility arising from any reliance placed on the content. Where the content includes any translated material, BMJ does not warrant the accuracy and reliability of the translations (including but not limited to local regulations, clinical guidelines, terminology, drug names and drug dosages), and is not responsible for any error and/or omissions arising from translation and adaptation or otherwise.

Open access This is an open access article distributed in accordance with the Creative Commons Attribution Non Commercial (CC BY-NC 4.0) license, which permits others to distribute, remix, adapt, build upon this work non-commercially, and license their derivative works on different terms, provided the original work is properly cited, appropriate credit is given, any changes made indicated, and the use is non-commercial. See: http://creativecommons.org/licenses/by-nc/4.0/.

ORCID iDs

Madelé van Dyk http://orcid.org/0000-0001-8721-6370 
Anna M Mc Laughlin http://orcid.org/0000-0002-5936-1877

Victoria Ziesenitz http://orcid.org/0000-0003-2836-4212

\section{REFERENCES}

1 National Cancer Institute. Seer cancer STAT facts: childhood leukaemia. Available: https://seer.cancer.gov/statfacts/html/childleuk. html

2 Namayandeh SM, Khazaei Z, Lari Najafi M, Najafi MN, et al. Global leukemia in children 0-14 statistics 2018, incidence and mortality and Human Development Index (HDI): GLOBOCAN sources and methods. Asian Pac J Cancer Prev 2020;21:1487-94.

3 Cooper SL, Brown PA. Treatment of pediatric acute lymphoblastic leukemia. Pediatr Clin North Am 2015;62:61-73.

4 Hossain MJ, Xie L, McCahan SM. Characterization of pediatric acute lymphoblastic leukemia survival patterns by age at diagnosis. $J$ Cancer Epidemiol 2014;2014:1-9.

5 Horton TM, Steuber CP. Overview of the treatment of acute lymphoblastic leukaemia in children and adolescents. UpToDate, 2018. Available: www.uptodate.com/contents/overview-of-thetreatment-of-acute-lymphoblastic-leukaemia-in-children-andadolescents

6 National Cancer Institute. Childhood Acute Myeloid Leukaemia/ Other Myeloid Malignancies Treatment (PDQ®)-Health Professional Version. Available: https://www.cancer.gov/types/leukaemia/hp/childaml-treatment-pdq

7 Queudeville M, Schlegel P, Heinz AT. Blinatumomab in pediatric patients with relapsed/refractory B-cell precursor acute lymphoblastic leukemia. Eur J Haematol 2020.
8 Kızılocak H, Okcu F. Late effects of therapy in childhood acute lymphoblastic leukemia survivors. Turk J Haem 2019;36:1-11.

9 Hawcutt DB, Cooney L, Oni L, et al. Precision dosing in children. Expert Rev Precis Med Drug Dev 2016;1:69-78.

10 Clarke W, Dasgupta A, eds. Clinical Challenges in Therapeutic Drug Monitoring: Special Populations, Physiological Conditions and Pharmacogenomics. 1st edn. Elsevier, 2016.

11 Groninger E, Proost JH, de Graaf SSN. Pharmacokinetic studies in children with cancer. Crit Rev Oncol Hematol 2004;52:173-97.

12 TGA. Australian product information glivec (imatinib) capsules \& film-coated tablets. Available: https://medicines.org.au/files/nvpglior. pdf

13 Soldin OP, Soldin SJ. Review: therapeutic drug monitoring in pediatrics. Ther Drug Monit 2002;24:1-8.

14 Paci A, Veal G, Bardin C, et al. Review of therapeutic drug monitoring of anticancer drugs part 1--cytotoxics. Eur J Cancer 2014;50:2010-9.

15 Gotta V, Widmer N, Decosterd LA, et al. Clinical usefulness of therapeutic concentration monitoring for imatinib dosage individualization: results from a randomized controlled trial. Cancer Chemother Pharmacol 2014;74:1307-19.

16 National, heart, lung and blood institute. Study quality assessment tools. Available: https://www.nhlbi.nih.gov/health-topics/studyquality-assessment-tools

17 Pluye P, Robert E, Cargo M, et al. Proposal: a mixed methods appraisal tool for systematic mixed studies reviews, 2011. Available: http://mixedmethodsappraisaltoolpublic.pbworks.com/w/file/fetch/ 84371689/MMAT\%202011\%20criteria\%20and\%20tutorial\%20201106-29updated2014.08.21.pdf 